\title{
Non Invasive Brain Stimulation to Improve Metaphoric Speech Processing in Schizophrenia: A Pilot Study
}

Michele Ribolsi ${ }^{\star}$, Giorgio Di Lorenzo, Cinzia Niolu, Vito Pinzone, Giulia Lisi, Emanuela Bianciardi, Anna Saya and Alberto Siracusano

Clinica Psichiatria, Dipartimento di Medicina dei Sistermi, Università degli Studi di Roma Tor Vergata, Rome, Italy

*Corresponding author: Michele Ribolsi, Clinica Psichiatrica, Dipartimento di Medicina dei Sistemi, Università Tor Vergata, Via Nomentana 1362, 00137, Rome, Italy, Tel: +39 06 41400356; Fax: +39 06 41400343; E-mail: michele.81@live.it

Received date: April 22, 2016; Accepted date: May 23, 2016; Published date: May 30, 2016

Copyright: @ 2016 Ribolsi M, et al. This is an open-access article distributed under the terms of the Creative Commons Attribution License, which permits unrestricted use, distribution, and reproduction in any medium, provided the original author and source are credited.

\begin{abstract}
Background: An underestimated feature of schizophrenia is the impaired processing of the metaphoric speech, mainly due to a reduced activation of the right hemisphere.
\end{abstract}

Objective/hypothesis: We investigated whether anodal tDCS over the right posterior superior temporal sulcus may improve the processing of metaphoric speech of schizophrenia patients (SCZ).

Methods: Ten SCZ subjects were randomly allocated to receive a single session of active tDCS or sham stimulation. Metaphoric speech processing was investigated through a discrimination task between literal or metaphoric expressions.

Results: tDCS improved significantly the reaction time but not the accuracy of the answers at the discrimination task compared to sham stimulation.

Conclusion: These preliminary results should be verified in larger studies with more sessions of tDCS.

Keywords: Schizophrenia; tDCS; Right superior temporal sulcus; Metaphor

\section{Introduction}

An intriguing hypothesis on schizophrenia is the possibility to interpret it as a semiotic disorder, with an impaired processing of the metaphoric speech $[1,2]$. In particular, various studies have shown that schizophrenia patients (SCZ) interpret metaphoric expressions more literally compared to healthy control subjects (HS) [3-5] and there is growing evidence also of impaired proverb comprehension [6-9]. Interestingly, a positive relation between such impairment and negative symptoms has been observed [5]. On the contrary, other studies have hypothesized a link with positive symptoms, supporting the hypothesis that delusions may represent a metaphoric representation of aspects of the world or of the self-expressed in a literal way $[3,10]$. According to fMRI investigations in HS, the right hemisphere and in particular the right superior temporal sulcus (STS) seems to play a pivotal role in processing metaphoric speech [11]. Interestingly, there is some evidence that SCZ exhibit less activation than HS in these areas during the comprehension of metaphoric vs. literal speech [12]. More recently, a reversed functional lateralization during novel metaphor processing has been found in SCZ, shoving an over activation in the left inferior frontal gyrus compared to HS [13]. A possible explanation is that the inefficient processing of novel metaphors in schizophrenia involves compensatory recruitment of additional brain regions, with an increased BOLD response in some left hemisphere areas than the right ones [13].

In this pilot study, starting from the hypothesis of a reduced activation of the right posterior STS during the metaphoric speech processing in SCZ, we investigated whether non-invasive brain stimulation by mean of anodal transcranial Direct Current Stimulation (tDCS) over the right STS compared to sham stimulation may improve the ability of these patients to process metaphoric speech.

\section{Materials and Methods}

\section{Patients}

Ten SCZ (seven males) patients were enrolled (mean age $34 \pm 5.7$ ). All patients were inpatients and were recruited from the Psychiatric Clinic at Tor Vergata University in Rome. Diagnosis was determined by means of consultation with physicians and the Structured Clinical Interview for Diagnostic and Statistical Manual of Mental Disorders, Fourth Edition. Patients were excluded from participating if they exhibited any neurological disorders, a history of head trauma, or if they met criteria for substance dependence within the previous 6 months or substance abuse within the month preceding testing. All patients were under neuroleptic treatment expressed in terms of chlorpromazine equivalents (mean dosage: $638 \pm 198$ )

\section{tDCS}

All the SCZ subjects were randomly allocated to receive active tDCS or sham stimulation. The interval between each session (active or sham) was one week. The tDCS was applied for $15 \mathrm{~min}$ through rectangular saline-soaked sponge electrodes $\left(50 \times 70 \mathrm{~mm}^{2}\right)$ with a battery-driven stimulator (CX-6650, Rolf Schneider Electronics, Gleichen, Germany). Stimulus intensity was set at $2 \mathrm{~mA}$. We applied anodal stimulation over the right posterior STS (corresponding to EEG 
coordinate T6) while the reference cathode electrode was placed over the contralateral shoulder.

\section{Discrimination task}

To investigate metaphoric speech processing, we used a discrimination task between literal or metaphoric or incongruous sentences. This task was conducted before and immediately after each session of both active and sham tDCS. The task is partly adapted from Iakimova et al. 80 incongruous sentences, 40 literal sentences, and 40 metaphors were presented to each patient before and after the tDCS session. Different sentences were used before and after the tDCS session. Each sentence was presented visually in a PC screen in four successive blocks (example: (1) Lucia (2) è una (3) bella (4) ragazza [(1) Lucia (2) is a (3) nice (4) girl)]. Each block was displayed for 200 $\mathrm{ms}$ followed by a white screen that was displayed for $350 \mathrm{~ms}$. The second sentence followed a white screen displayed for $2200 \mathrm{~ms}$. During this interval, each subject was asked to judge whether the sentence made sense or not. The left button corresponded to a "yes" response and the right button to a "no" response. A training phase was conducted before the task. The metaphoric expressions were commonly used Italian metaphors with an average familiarity of $90 \%$ (rated by $20 \mathrm{HS}$ not included in the study).

\section{Results}

Because of the small size of the sample, nonparametric statistics (Mann-Whitney U tests) were used to compare the performance at the discrimination task following tDCS and sham stimulation. tDCS improved significantly the reaction time of the answers at the discrimination task $(\mathrm{U}=55, \mathrm{p}=0.015)$ compared to sham stimulation $(\mathrm{U}=25.5, \mathrm{p}=0.89)$ (Figure 1). On the contrary, neither active tDCS $(\mathrm{U}=38, \mathrm{p}=0.59)$ nor sham stimulation $(\mathrm{U}=36.5 ; \mathrm{p}=0.63)$ improved significantly the accuracy at the discrimination task.

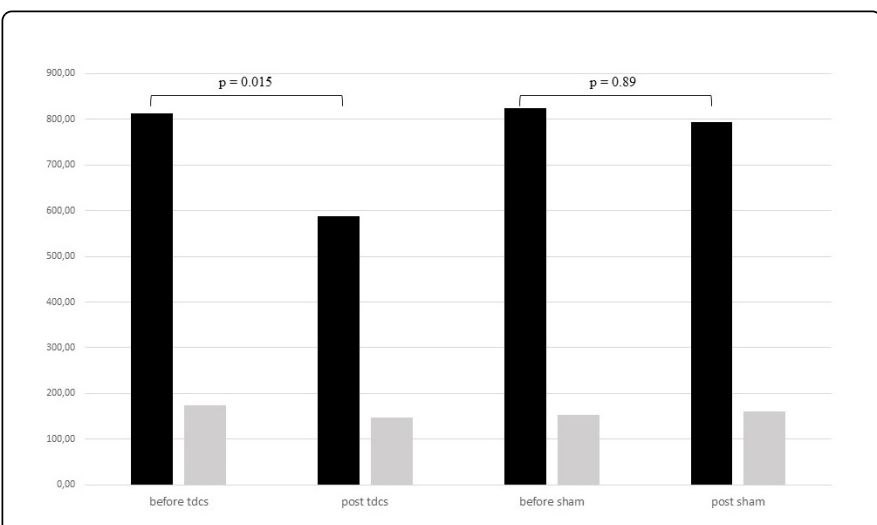

Figure 1: Reaction time (ms) following active and sham stimulation Black bars represent the mean reaction time observed in the four conditions (before tDCS, after tDCS, before sham and after sham stimulation), while the grey bars represent the related standard deviations.

\section{Conclusion}

In our experiment both tDCS and sham stimulation were well tolerated. This is in line with previous reports which described only mild or transient side effects across a wide range of SCZ patients who underwent tDCS $[14,15]$.

The main finding of this study is that that anodal tDCS over the right STS compared to sham stimulation improved significantly the reaction time (RT) of the answers during a task of discrimination between literal, incongruous and metaphoric sentences. A possible explanation of this improvement is that there is a likelihood of practice effects affecting the results of the study. However, we used different sentences with a different order of presentation (literal, metaphoric or incongruous) before and after the tDCS session. Moreover, the short duration of the stimulus $(200 \mathrm{~ms})$ and of the interstimulus interval $(350 \mathrm{~ms})$ should minimize the risk of practice effects. We hypothesize that such improvement may have some benefits in terms of disease management, as the impairment in metaphoric speech is related to positive symptoms, in particular to the foundation of delusions [10]. However, further research should be necessary to evaluate the effects of multiple tDCS sessions on the processing of metaphoric speech and its impact on psychotic symptoms. Despite improvements in reaction time, we didn't find any change of accuracy of the answers was observed after both sham and active stimulation. A possible explanation of these results may be that active stimulation, while improving the RT, does not improve the accuracy of the answers because of the intrinsic difficulty of the task for medicated SCZ patients. To this regard, it is noteworthy that the task used in this study was characterized by a short duration of the stimulus $(200 \mathrm{~ms})$ and of the interstimulus interval ( $350 \mathrm{~ms})$.

Therefore, it should be hypothesized that in this pilot study the active stimulation provide only subclinical effect (as confirmed by the improvement of the RT) that could be verified through EEG or fMRI recordings in further studies. Another possibility is that metaphoric speech processing involves the recruitment of a more complex network and the selective stimulation of a brain area is not sufficient to produce an effect in terms of accuracy of answers. Finally, it should be useful to investigate in a larger sample of patients also the neurophysiological effect of more sessions of active stimulation compared to sham stimulation by mean of EEG or fMRI recordings.

\section{References}

1. Harrod JB (1986) Schizophrenia as a semiotic disorder. Schizophr Bull 12: 12-19.

2. Mitchell RL, Crow TJ (2005) Right hemisphere language functions and schizophrenia: The forgotten hemisphere? Brain 128: 963-978.

3. Elvevåg B, Helsen K, De Hert M, Sweers K, Storms G (2011) Metaphor interpretation and use: A window into semantics in schizophrenia. Schizophr Res 133: 205-211.

4. Mo S, Su Y, Chan RC, Liu J (2008) Comprehension of metaphor and irony in schizophrenia during remission: The role of theory of mind and IQ. Psychiatry Res 157: 21-29.

5. Mossaheb N, Aschauer HN, Stoettner S, Schmoeger M, Pils N, et al. (2014) Comprehension of metaphors in patients with schizophreniaspectrum disorders. Compr Psychiatry 55: 928-937.

6. Rapp AM, Langohr K, Mutschler DE, Wild B (2014) Irony and proverb comprehension in schizophrenia: Do female patients "dislike" ironic remarks? Schizophr Res Treatment 2014: 841086.

7. Haas MH, Chance SA, Cram DF, Crow TJ, Luc A, et al. (2015) Evidence of pragmatic impairments in speech and proverb interpretation in schizophrenia. J Psycholinguist Res 44: 469-483.

8. Thoma P, Hennecke M, Mandok T, Wähner A, Brüne M, et al. (2009) Proverb comprehension impairments in schizophrenia are related to executive dysfunction. Psychiatry Res 170: 132-139. 
Citation: Ribolsi M, Lorenzo GD, Niolu C, Pinzone V, Lisi G, et al. (2016) Non Invasive Brain Stimulation to Improve Metaphoric Speech Processing in Schizophrenia: A Pilot Study. J Neurol Neurophysiol 7: 375. doi:10.4172/2155-9562.1000375

Page 3 of 3

9. Brüne $\mathrm{M}$, Bodenstein L (2005) Proverb comprehension reconsidered--'theory of mind' and the pragmatic use of language in schizophrenia. Schizophr Res 75: 233-239.

10. Rhodes JE, Jakes S (2004) The contribution of metaphor and metonymy to delusions. Psychol Psychother 77: 1-17.

11. Pobric G, Mashal N, Faust M, Lavidor M (2008) The role of the right cerebral hemisphere in processing novel metaphoric expressions: A transcranial magnetic stimulation study. J Cogn Neurosci 20: 170-181.

12. Kircher TT, Leube DT, Erb M, Grodd W, Rapp AM (2007) Neural correlates of metaphor processing in schizophrenia. Neuroimage 34: 281-289.
13. Mashal N, Vishne T, Laor N, Titone D (2013) Enhanced left frontal involvement during novel metaphor comprehension in schizophrenia: Evidence from functional neuroimaging. Brain Lang 124: 66-74.

14. Fregni F, Nitsche MA, Loo CK, Brunoni AR, Marangolo P, et al. (2015) Regulatory considerations for the clinical and research use of transcranial direct current stimulation (tDCS): Review and recommendations from an expert panel. Clin Res Regul Aff 32: 22-35.

15. Mondino M, Brunelin J, Palm U, Brunoni AR, Poulet E, et al. (2015) Transcranial direct current stimulation for the treatment of refractory symptoms of schizophrenia. Current evidence and future directions. Curr Pharm Des 21: 3373-3383. 